\title{
Experiência Multicêntrica na América do Sul no Fechamento Perventricular da Comunicação Interventricular Muscular Congênita
}

\author{
Carlos Augusto Cardoso Pedra ${ }^{1,2}$, Simone R. F. Pedra ${ }^{1,2}$, Paulo Chaccur ${ }^{1,2}$, Marcelo B. Jatene', \\ Maria Virginia T. Santana ${ }^{2}$, Ana Luíza Guerra ${ }^{2}$, Cristiane Pessotti ${ }^{1}$, leda B. Jatene', \\ Ricardo Fonseca Martins², Guillermo Villoria ${ }^{3}$, Isac Tueti ${ }^{3}$, Justo Santiago ${ }^{4}$, \\ Igor Donis ${ }^{3}$, Valmir F. Fontes ${ }^{1,2}$
}

\section{RESUMO}

Fundamentos: $O$ fechamento perventricular das comunicações interventriculares (CIVs) musculares tornou-se uma alternativa terapêutica atraente em pequenos lactentes com grandes defeitos. Entretanto, a reprodutibilidade desse método em vários centros mundiais ainda é uma questão em aberto. Método: Relatamos uma experiência multicêntrica na América do Sul com tal abordagem. No período de julho de 2007 a maio de 2009, nove pacientes nãoconsecutivos (idade e peso médios de 6 meses e $5,5 \mathrm{~kg}$, respectivamente) foram submetidos a procedimento no centro cirúrgico, sob monitoração da ecocardiografia transesofágica, utilizando-se dispositivos Amplatzer. Todos os pacientes, com exceção de um, apresentavam insuficiência cardíaca e graus variados de hipertensão pulmonar. Quatro pacientes eram portadores de coartação da aorta e um tinha sido submetido a bandagem da artéria pulmonar com 6 meses de vida, todos reparados cirurgicamente na mesma sessão. Oito pacientes possuíam defeitos únicos (seis no meio do septo e dois, apicais), medindo $10,3 \pm 3,7 \mathrm{~mm}$, e um paciente apresentava dois defeitos apicais que necessitaram do implante de dois dispositivos. Resultados: Dez dispositivos foram implantados com sucesso (tamanho médio de $12 \mathrm{~mm}$ ), sendo dois fixados na parede livre do

\author{
ABSTRACT \\ Perventricular Closure of Congenital Muscular \\ Ventricular Septal Defect: Multicenter \\ Experience in South America
}

Background: Perventricular closure of muscular ventricular septal defects (VSD) has become an attractive treatment modality for infants. However, its reproducibility worldwide remains to be seen. Methods: We report a multicenter experience in South America. From July, 2007 to May, 2009, nine non-consecutive patients (median age and weight were 6 months and $5.5 \mathrm{~kg}$, respectively) underwent the procedure in the operating room under transesophageal echocardiographic guidance using Amplatzer devices. All patients but one were in congestive heart failure and had pulmonary arterial hypertension. Four patients had coarctation of the aorta and one was submitted to pulmonary artery banding at 6 months of life, which were all repaired at the same session. Eight patients had single defects (six mid-muscular, two apical) measuring $10.3 \pm 3.7 \mathrm{~mm}$ and one patient had multiple apical defects that required two devices. Results: Ten devices were implanted successfully (median size: $12 \mathrm{~mm}$ ), and two had to be sutured to the right ventricular wall with a surgical suture. One patient

Este artigo foi apresentado como pôster no Congresso da Sociedade Latino-Americana de Cardiologia Intervencionista (SOLACI), no Rio de Janeiro, Brasil, em junho de 2009, e ganhou o prêmio de melhor trabalho in situ, julgado pela Comissão Científica desse congresso. Foi também apresentado como pôster no Congresso Mundial de Cardiologia Pediátrica, em Cairns, Austrália, em julho de 2009.

1 Hospital do Coração da Associação Sanatório Sírio - São Paulo, SP, Brasil.

2 Instituto Dante Pazzanese de Cardiologia - São Paulo, SP, Brasil.

${ }^{3}$ Hospital Cardiológico Infantil Latino-Americano Dr. Gustavo Uchoa Mendez - Caracas, Venezuela.

${ }^{4}$ Hospital Universitário de Los Andes - Mérida, Venezuela.

Correspondência: Carlos A. C. Pedra. Instituto Dante Pazzanese de Cardiologia. Av. Dr. Dante Pazzanese, 500 - 14º andar - São Paulo, SP, Brasil - CEP 04012-180

E-mail: cacpedra@uol.com.br

Recebido em: 10/6/2009 • Aceito em: 4/9/2009 
Pedra CAC, et al. Experiência Multicêntrica na América do Sul no Fechamento Perventricular da Comunicação Interventricular Muscular Congênita. Rev Bras Cardiol Invas. 2009;17(3):386-97.

ventrículo direito. Um paciente apresentou morfologia de bloqueio de ramo direito e outro, de bloqueio de ramo esquerdo após o implante. Em um paciente com CIV de $14 \mathrm{~mm}$ com borda póstero-inferior mais curta, a porção inferior de um dispositivo de $16 \mathrm{~mm}$ sofreu prolapso através do defeito para o ventrículo direito, necessitando retirada cirúrgica e ventriculosseptoplastia com retalho pericárdico. Após seguimento médio de um ano, todos os oito pacientes (incluindo o com CIVs múltiplas) apresentavam oclusão do defeito e retorno do ventrículo esquerdo às dimensões normais. Conclusão: A oclusão perventricular das CIVs musculares foi factível, reprodutível, de modo geral segura e bem efetiva nesta experiência multicêntrica. Defeitos maiores podem necessitar de superdimensionamento do dispositivo e/ou fixação com suturas cirúrgicas para adequada estabilização. Maior número de pacientes e período de seguimento mais longo são necessários para conclusões mais definitivas.

DESCRITORES: Comunicação interventricular. Cateterismo cardíaco. Próteses e implantes.
A comunicação interventricular muscular é um dos tipos de comunicação interventricular de origem congênita, podendo estar localizada nas regiões trabecular, apical ou anterior ${ }^{1,2}$. Por definição, está circundada por tecido muscular ao longo de todo o defeito e geralmente fica distante do nó atrioventricular ${ }^{1,2}$. Quando pequena, a história natural demonstra boas chances de oclusão espontânea até quatro ou cinco anos de idade ${ }^{1,2}$. Seu tratamento está indicado quando causa insuficiência cardíaca no primeiro ano de vida ou, após essa idade, quando há repercussão hemodinâmica, caracterizada pelo aumento das dimensões do ventrículo esquerdo à ecocardiografia ${ }^{1,2}$.

O tratamento percutâneo da comunicação interventricular muscular apresenta ótimos resultados com as próteses intracardíacas de última geração ${ }^{3-10}$, inclusive em nosso meio ${ }^{11,12}$, sendo o método de escolha para o fechamento desse tipo de defeito, na maioria dos casos, nos maiores centros mundiais. Entretanto, a baixa faixa etária é considerada um fator de risco para complicações decorrentes da oclusão percutânea da comunicação interventricular muscular na sala de cateterismo ${ }^{5,10}$. Para neutralizar esse fator, alguns autores passaram a realizar a oclusão da comunicação interventricular muscular com próteses implantadas pela via perventricular com toracotomia mediana ${ }^{13-19}$. Tal abordagem, introduzida por Amin et al. ${ }^{13}$, demonstrou-se segura e eficaz em experiências subsequentes, porém com número limitado de pacientes e em poucos serviços. Em decorrência da escassez de publicações abordando esse método terapêutico, sua reprodutibilidade em diferentes centros ainda se mantém como uma questão em aberto.

Este artigo relata uma experiência multicêntrica na América do Sul com o fechamento perventricular da developed right bundle branch block and another one developed left bundle branch block. In one patient with a $14 \mathrm{~mm}$ defect, the inferior portion of a $16 \mathrm{~mm}$ device prolapsed through the inferior rim of the defect towards the right ventricle, requiring surgical removal with patch closure of the mid-muscular VSD. After a median one year follow-up, all the eight remaining patients, including the one with multiple VSDs, had complete closure of the defects with normal left ventricular size. Conclusion: Perventricular closure of muscular VSDs seems to be reproducible, feasible, relatively safe and effective in this multicenter study. Large defects may require device oversizing or suture fixation for adequate stabilization. More patients and longer follow-up are needed for stronger conclusions.

DESCRIPTORS: Heart septal defects, ventricular. Heart catheterization. Prostheses and implants.

comunicação interventricular muscular em pequenos lactentes e crianças, analisando a viabilidade, a segurança e a eficácia do método.

\section{MÉTODO}

\section{Desenho do estudo}

Trata-se de estudo prospectivo longitudinal observacional de uma coorte de lactentes e crianças com idade inferior a dois anos, submetidos a oclusão perventricular da comunicação interventricular muscular congênita com próteses intracardíacas de forma nãoconsecutiva em múltiplos centros. Os responsáveis legais pelos pacientes foram adequadamente informados sobre os riscos e benefícios do procedimento e assinaram o termo de consentimento. O estudo foi aprovado pela comissão de ética local.

\section{Critérios de inclusão}

1. Lactentes com menos de $8 \mathrm{~kg}$, de ambos os sexos, com diagnóstico clínico e ecocardiográfico de comunicações interventriculares musculares únicas ou múltiplas, de localização variada (trabecular no meio do septo, apical ou anterior), com repercussão hemodinâmica, caracterizadas pela presença de insuficiência cardíaca de difícil controle e/ou aumento significativo do ventrículo esquerdo (maior que dois desvios padrão da normalidade para a idade).

2. Lactentes e crianças com comunicações interventriculares musculares com repercussão hemodinâmica, associadas à presença de lesões extracardíacas, como coartação da aorta, com indicação de abordagem cirúrgica sem circulação extracorpórea.

3. Lactentes e crianças com comunicações interventriculares musculares com repercussão hemodinâ- 
Pedra CAC, et al. Experiência Multicêntrica na América do Sul no Fechamento Perventricular da Comunicação Interventricular Muscular Congênita. Rev Bras Cardiol Invas. 2009;17(3):386-97.

mica, previamente submetidas a bandagem da artéria pulmonar, com indicação de retirada da bandagem após oclusão da comunicação interventricular.

\section{Critérios de exclusão}

1. Comunicações interventriculares de outros tipos, incluindo a comunicação interventricular perimembranosa, a de via de entrada e de via de saída, também chamada de duplamente relacionada.

2. Comunicações interventriculares musculares com ventrículo esquerdo de dimensões normais.

3. Doenças cardíacas associadas com necessidade de intervenção cirúrgica, com uso de circulação extracorpórea concomitante ao procedimento.

\section{Doenças infecciosas ativas.}

5. Alergia comprovada ao níquel.

6. Comunicações interventriculares musculares de anatomia desfavorável para tratamento perventricular, caracterizadas por comunicações interventriculares de grande tamanho, nas quais se previa uso de prótese com discos de diâmetro excessivamente grande para as dimensões dos ventrículos e/ou muito próximas das valvas atrioventriculares ou semilunares. Esse item foi definido por meio de ecocardiografia transtorácica e/ou transesofágica antes ou durante o procedimento, realizada por profissional experiente na seleção dos pacientes.

7. Recusa em assinar o termo de consentimento.

\section{Avaliação clínica pré-procedimento}

Os pacientes foram submetidos a avaliação clínica rotineira com obtenção de exames laboratoriais, incluindo eletrocardiografia, radiografia de tórax, ecocardiografia transtorácica, hemograma, coagulograma, eletrólitos, glicemia, avaliação da função renal, tipagem sanguínea e urina tipo I.

\section{Dispositivos}

Neste estudo, foram utilizados dispositivos Amplatzer (AGA Medical Corporation, Plymouth, Estados Unidos). O dispositivo para fechamento da comunicação interventricular muscular é uma prótese feita de malha de fios ultrafinos de nitinol, composta de dois discos conectados por cintura central, extensivamente descrita em publicações anteriores ${ }^{3-12}$. Selecionou-se um dispositivo cuja cintura central, que possui diâmetros de $4 \mathrm{~mm}$ a $18 \mathrm{~mm}$, fosse $1 \mathrm{~mm}$ a $3 \mathrm{~mm}$ maior que o maior diâmetro do defeito medido no final da diástole. Tal prótese requer o uso de bainhas de baixo perfil para o implante (entre $6 \mathrm{~F}$ e $9 \mathrm{~F}$ ). Esses dispositivos possuem registro na Agência Nacional de Vigilância Sanitária (Anvisa) e estão liberados para uso clínico no Brasil. Também estão liberados para uso clínico pelo Food and Drug Administration (FDA), dos Estados
Unidos, desde agosto de 2007, e possuem o CE Mark europeu.

\section{Procedimentos}

Depois de observado período de jejum de oito horas, os pacientes foram levados ao centro cirúrgico e submetidos ao procedimento sob anestesia geral, com monitoração pela ecocardiografia transesofágica. Não foram utilizados equipamentos portáteis de radioscopia e angiografia. Após toracotomia mediana para exposição do coração, sulfato de heparina foi administrado na dose de $100 \mathrm{UI} / \mathrm{kg}$ juntamente com cefalotina (30 mg/kg). Sob orientação da ecocardiografia transesofágica, foi identificado o melhor local de punção da parede livre do ventrículo direito, a fim de se obter o trajeto mais retilíneo para cruzar a comunicação interventricular com um fio-guia (Figura 1). Após a realização de uma sutura em bolsa no local mais apropriado da parede do ventrículo direito, esta foi puncionada com uma agulha de calibre 17-18 e um guia de 0,038 polegada com ponta em "J" foi avançado pela comunicação interventricular para o ventrículo esquerdo, sob monitoração ecocardiográfica (Figura 2). A agulha foi retirada e um introdutor de perfil apropriado para o tamanho do dispositivo a ser implantado foi avançado sobre o guia pela parede do ventrículo direito até a cavidade livre do ventrículo esquerdo (Figura 3). A fim de evitar lesões e perfurações na parede posterior do ventrículo esquerdo, a distância entre as paredes anterior do ventrículo direito e posterior do ventrículo esquerdo foi determinada pela ecocardiografia na projeção 4 câmaras (Figura 4). O introdutor foi então marcado com fios de sutura de algodão, levando-se em conta essa mesma distância desde sua extremidade (Figura 3). Após estabilização do introdutor no corpo do ventrículo esquerdo, o guia e o dilatador foram retirados e um dispositivo de tamanho apropriado para o defeito foi avançado e implantado sob monitoração exclusiva da ecocardiografia, segundo técnicas já descritas anteriormente ${ }^{13-19}$ (Figura 5). Nos casos de comunicações interventriculares apicais, se não houvesse espaço para reconfiguração completa do disco de lado direito, o pino fêmea do dispositivo era fixado e suturado na parede externa do ventrículo direito (Figura 6). Nos casos de múltiplos defeitos, o defeito residual foi abordado de forma semelhante ao anterior, com implante de nova prótese. O posicionamento final do dispositivo e a presença de fluxos residuais foram avaliados pela ecocardiografia. Confirmado o posicionamento correto, a prótese foi liberada e novas avaliações ecocardiográficas foram realizadas. O introdutor foi retirado da parede livre do ventrículo direito, que foi reparada pela sutura em bolsa previamente realizada.

No caso da presença concomitante de coartação da aorta ou bandagem do tronco da artéria pulmonar, tais lesões foram reparadas pelo cirurgião seguindo as técnicas cirúrgicas clássicas, sem a utilização da circulação extracorpórea. 
Pedra CAC, et al. Experiência Multicêntrica na América do Sul no Fechamento Perventricular da Comunicação Interventricular Muscular Congênita. Rev Bras Cardiol Invas. 2009;17(3):386-97.
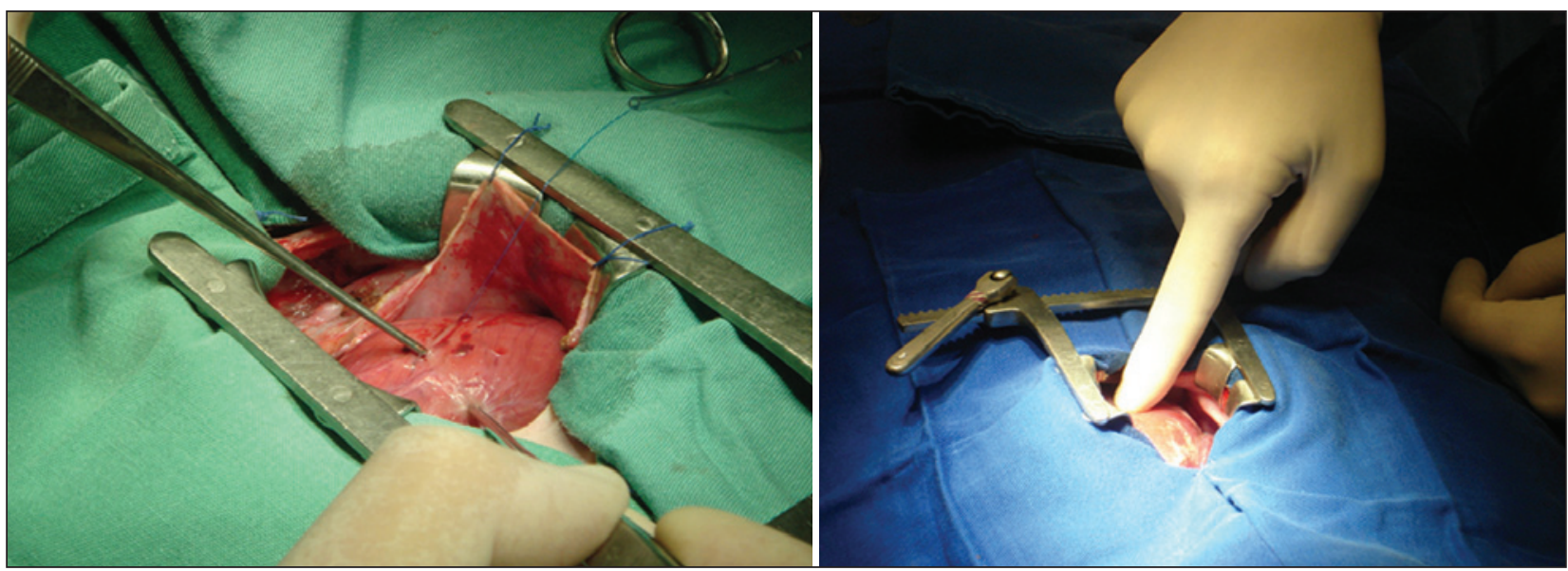

Figura 1 - Abertura do tórax e identificação do local de entrada. Após toracotomia mediana e fixação do saco pericárdico, utilizam-se pinças cirúrgicas (à esquerda) ou o dedo indicador (à direita) para compressão externa da parede livre do ventrículo direito, com o intuito de identificar o melhor local para punção e fixação da bainha de implante. O local abaulado e sua relação com a comunicação interventricular são averiguados pela ecocardiografia transesofágica (ver Figura 4)
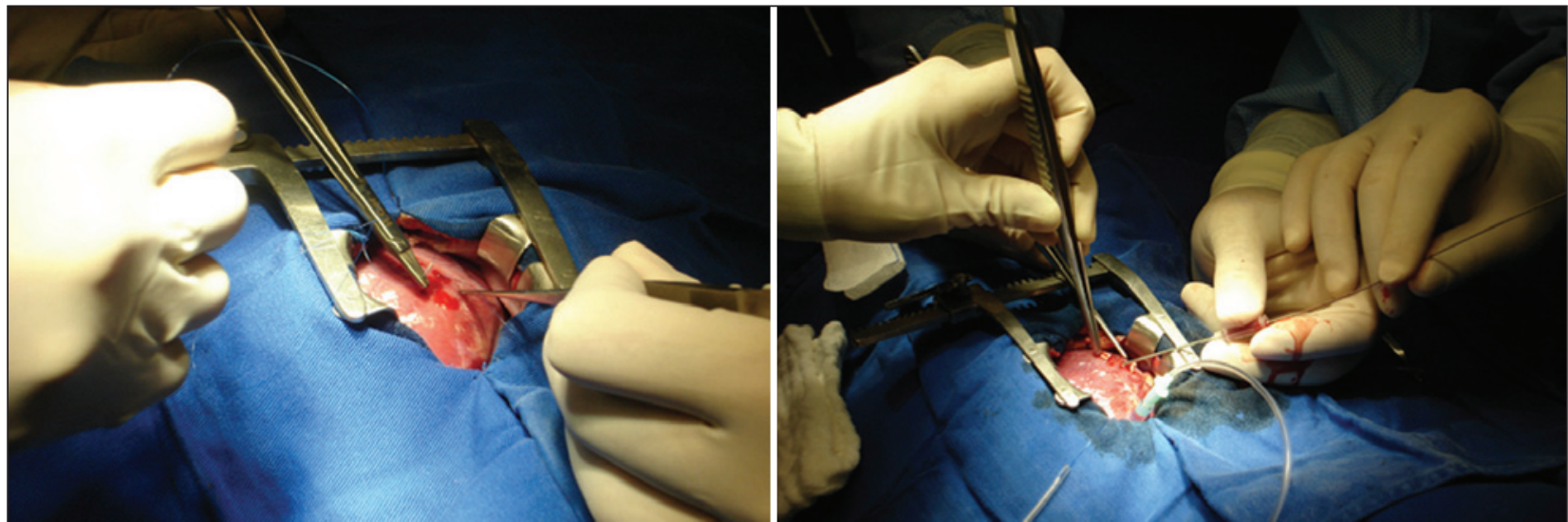

Figura 2 - Sutura em bolsa e punção na parede livre do ventrículo direito. À esquerda, sutura em bolsa realizada pelo cirurgião na parede livre do ventrículo direito após identificação do melhor local de punção. À direita, agulha padrão utilizada para punção da parede livre do ventrículo direito, permitindo a passagem do fio-guia do ventrículo direito para o esquerdo.
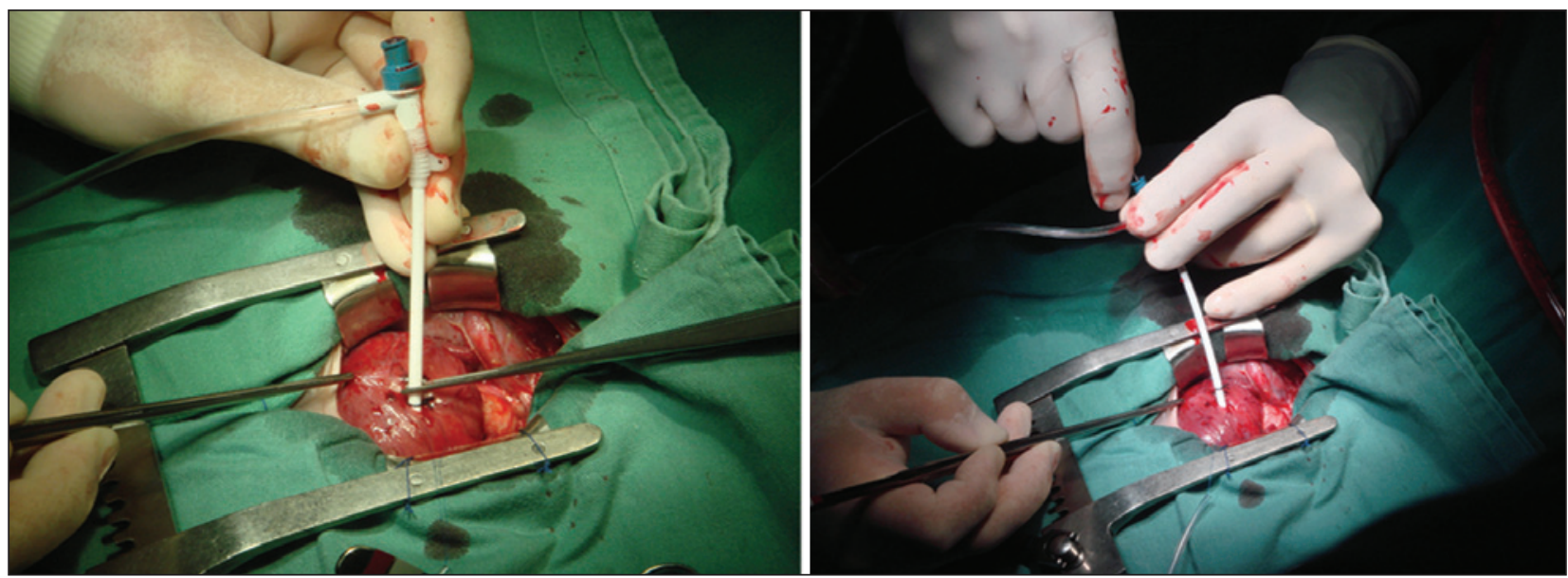

Figura 3 - Aspecto da bainha posicionada na parede livre ventricular. À esquerda, bainha $8 \mathrm{~F}$ posicionada pela parede livre do ventrículo direito. Nota-se a linha de sutura perto da parede ventricular. À direita, após a transferência da prótese para a bainha, o implante é realizado com movimentos delicados, empurrando-se o cabo liberador e puxando-se levemente a bainha. 
Pedra CAC, et al. Experiência Multicêntrica na América do Sul no Fechamento Perventricular da Comunicação Interventricular Muscular Congênita. Rev Bras Cardiol Invas. 2009;17(3):386-97.
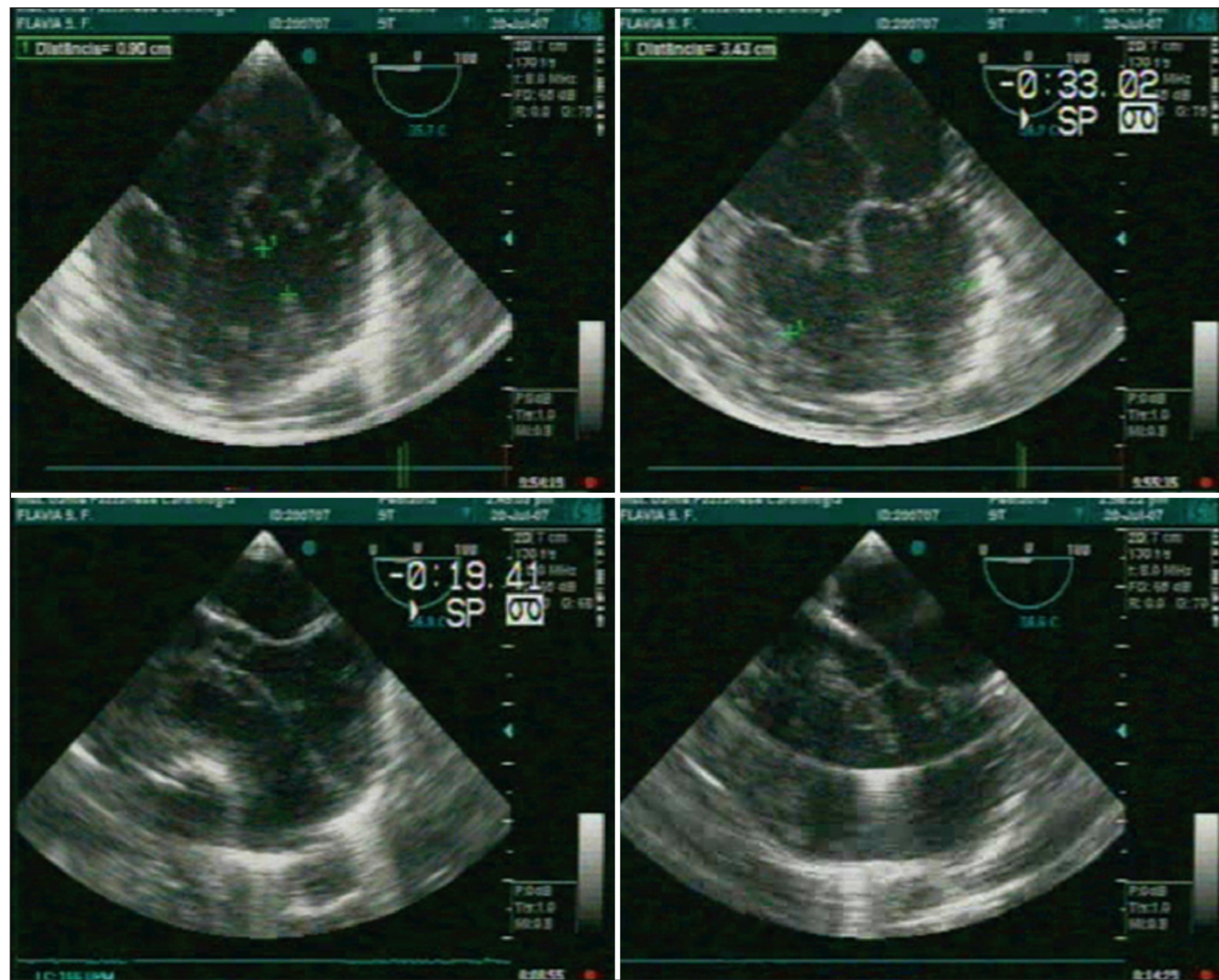

Figura 4 - Implante perventricular. Ecocardiografia transesofágica intraoperatória. Projeção 4 câmaras. No painel superior esquerdo, comunicação interventricular muscular trabecular única ampla $(9 \mathrm{~mm})$ em porções medianas do septo. No painel superior direito, medida da distância entre a parede anterior do ventrículo direito e a parede posterior do ventrículo esquerdo $(3,5 \mathrm{~cm})$. No painel inferior esquerdo, abaulamento interno da parede anterior do ventrículo direito causado pela compressão externa realizada pelo dedo indicador para avaliação do melhor local para punção com a agulha. E no painel inferior direito, após punção da parede livre do ventrículo direito, um guia 0,038 polegada é avançado pela comunicação interventricular até o ventrículo esquerdo.

\section{Avaliação pós-procedimento}

Após o fechamento do tórax por planos, de maneira usual, os pacientes foram transferidos para unidade de terapia intensiva para manejo pós-operatório rotineiro. A necessidade de uso e doses das medicações anticongestivas e a velocidade de retirada da ventilação mecânica foram reavaliadas caso a caso. A ecocardiografia transtorácica foi empregada nos primeiros dias após o procedimento para avaliação do posicionamento da prótese e presença de possíveis fluxos residuais. Aqueles que estavam bem clinicamente e sem complicações receberam alta hospitalar com orientação para tomarem aspirina (dose de 3-5 mg/kg/dia) por seis meses e para seguirem as recomendações clássicas para profilaxia de endocardite quando necessário. O seguimento clínico constou de exame físico, realização de radiografia de tórax (Figura 7), eletrocardiograma, e ecocardiografia transtorácica com Doppler em cores com 1, 6 e 12 meses após implante e a partir daí anualmente.

\section{Análise estatística}

Os valores são expressos em frequências e porcentagens, média e desvio padrão ou mediana e variação, conforme distribuição da amostra.

\section{RESULTADOS}

As características da população, os defeitos, os procedimentos e os desfechos ecocardiográficos estão descritos na Tabela 1. 
Pedra CAC, et al. Experiência Multicêntrica na América do Sul no Fechamento Perventricular da Comunicação Interventricular Muscular Congênita. Rev Bras Cardiol Invas. 2009;17(3):386-97.

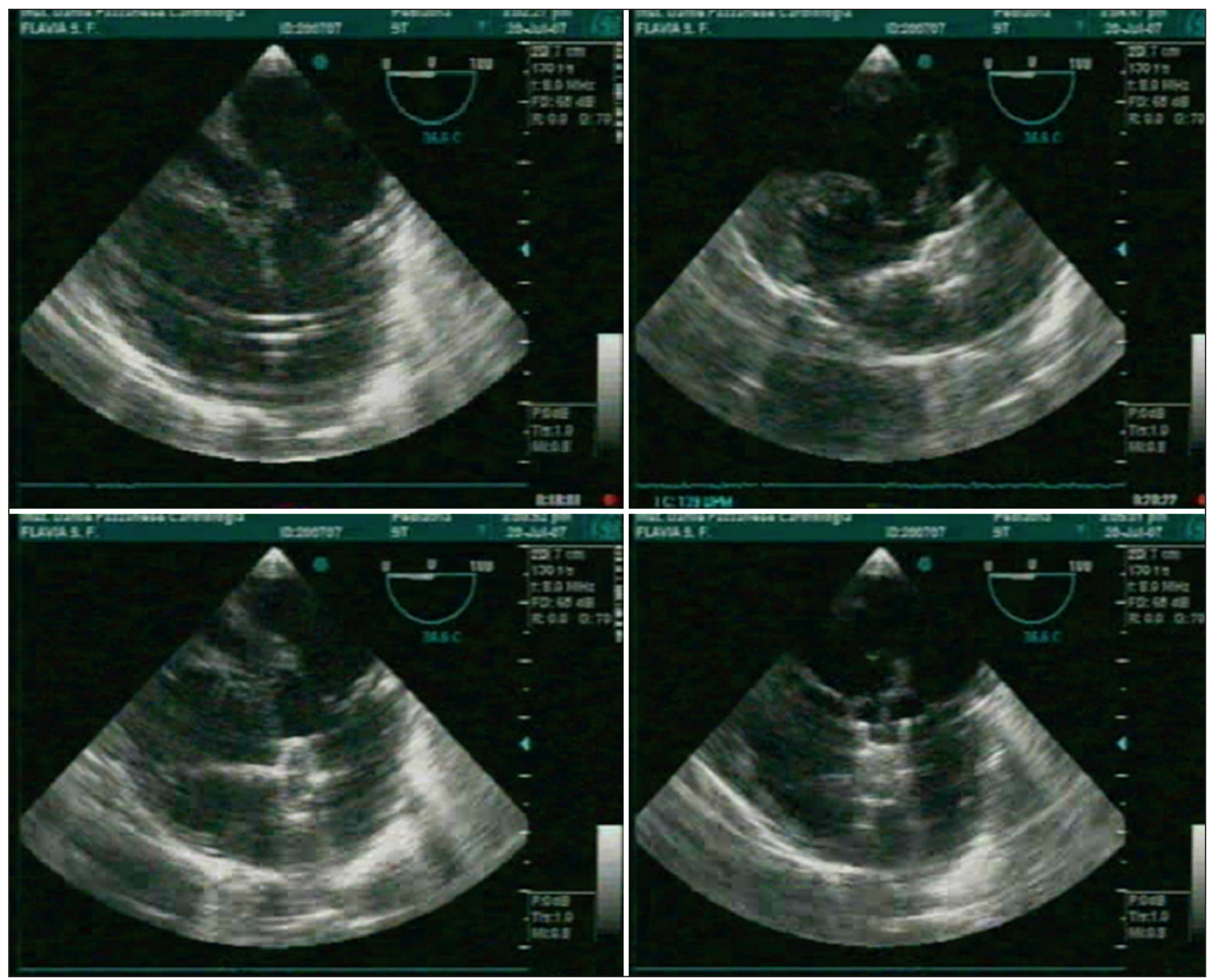

Figura 5 - Implante perventricular. Ecocardiografia transesofágica intraoperatória. Projeção 4 câmaras. No painel superior esquerdo, bainha $8 \mathrm{~F}$ avançada sobre o guia até o ventrículo esquerdo, sem chegar à parede posterior. No painel superior direito, disco esquerdo e cintura central da prótese $12 \mathrm{~mm}$ abertos no ventrículo esquerdo. No painel inferior esquerdo, disco esquerdo em contato com o septo interventricular. E no painel inferior direito, posicionamento adequado da prótese, abraçando o septo interventricular, após a abertura do disco do lado direito.

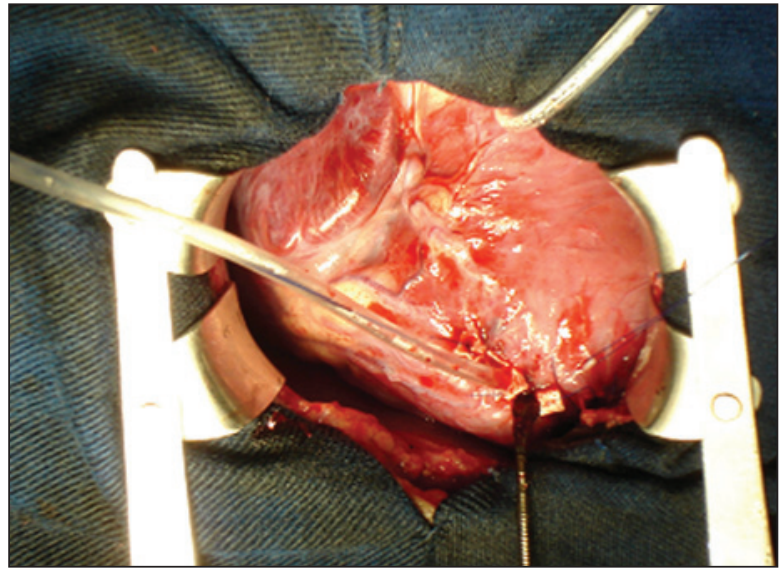

Figura 6 - Nota-se parte do disco direito exteriorizado e fixado por suturas com calços no ápice do ventrículo direito. O parafuso fêmea da prótese Amplatzer ainda está conectado ao cabo liberador.

\section{População e características dos defeitos}

No período de julho de 2007 a maio de 2009, foram realizados nove procedimentos em nove pacientes não-consecutivos em três centros: dois em São Paulo, SP e um em Caracas, Venezuela. A mediana da idade foi de 6 meses (45 dias a 24 meses) e do peso, de $5,5 \mathrm{~kg}(3,5 \mathrm{~kg}$ a $12 \mathrm{~kg})$. Todos os pacientes, exceto um (que havia sido submetido a bandagem do tronco da artéria pulmonar com 6 meses de vida), encontravam-se em insuficiência cardíaca de difícil controle clínico e apresentavam hipertensão arterial pulmonar. Em relação às enfermidades associadas, quatro pacientes eram portadores de coartação da aorta grave. Como já foi mencionado, um paciente de 2 anos e $12 \mathrm{~kg}$ havia sido submetido a bandagem do tronco da artéria pulmonar com 6 meses de vida e encontrava-se bem clinicamente. 
Pedra CAC, et al. Experiência Multicêntrica na América do Sul no Fechamento Perventricular da Comunicação Interventricular Muscular Congênita. Rev Bras Cardiol Invas. 2009;17(3):386-97.
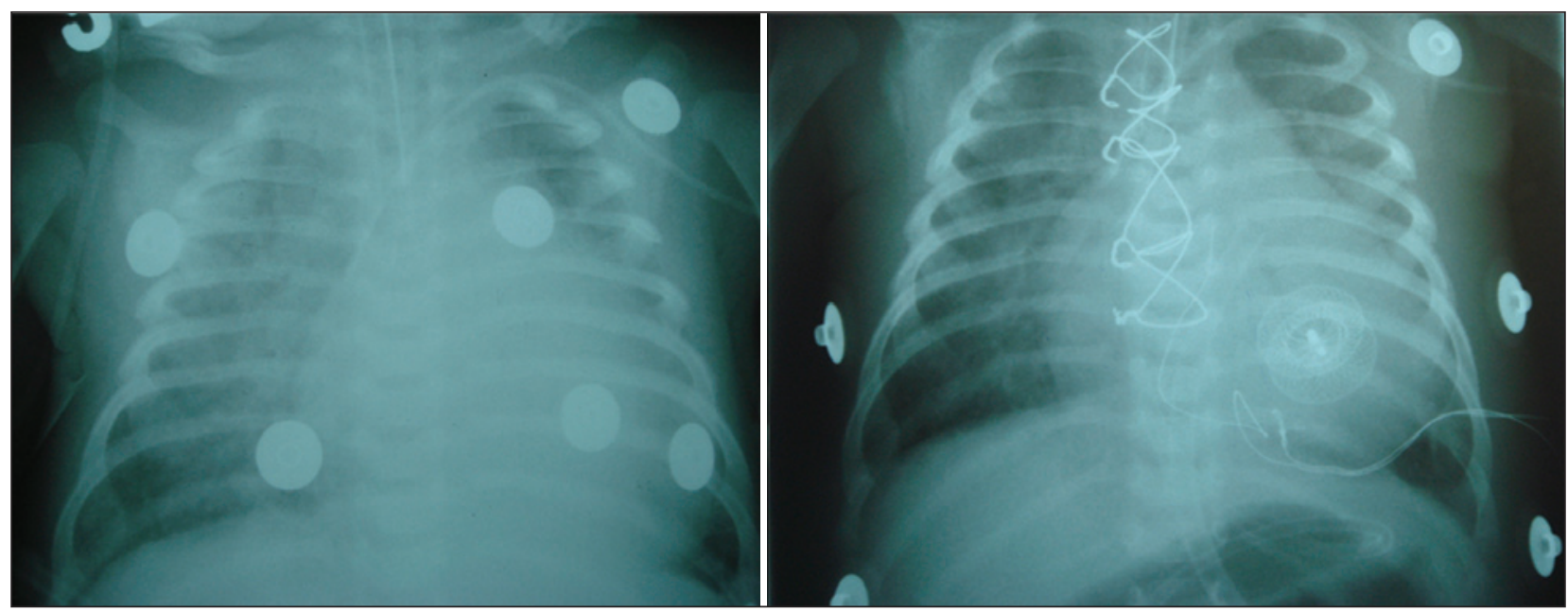

Figura 7 - Aspectos radiológicos do primeiro paciente da casuística. À esquerda, área cardíaca aumentada e hiperfluxo pulmonar evidente. À direita, após oclusão perventricular do defeito na porção trabecular média do septo, nota-se nítida redução do tamanho da área cardíaca e da trama vascular pulmonar. A prótese pode ser facilmente identificada no meio da porção esquerda da silhueta cardíaca.

TABELA 1

Características dos pacientes, defeitos, procedimentos e desfechos ecocardiográficos

\begin{tabular}{|c|c|c|c|c|c|c|c|c|c|}
\hline Paciente & Idade & Peso (kg) & $\begin{array}{l}\text { Local da } \\
\text { CIV }\end{array}$ & $\begin{array}{c}\text { Tamanho da } \\
\text { CIV (mm) }\end{array}$ & $\begin{array}{c}\text { Defeitos } \\
\text { associados }\end{array}$ & Prótese & Complicações & $\begin{array}{c}\text { Fluxo } \\
\text { residual } \\
\text { imediato }\end{array}$ & $\begin{array}{c}\text { Fluxo } \\
\text { residual } \\
\text { tardio }\end{array}$ \\
\hline 1 & 45 dias & 3,5 & Trabecular média & 9 & $\mathrm{CoA}$ & 12 & Transfusão & Não & Não \\
\hline 2 & 5 meses & 5 & Apical & 12 & - & 14 & Transfusão & Não & Não \\
\hline 3 & 13 meses & 7,9 & Apicais & 4 e 6 & - & 6 e 8 & $\begin{array}{c}\text { Transfusão } \\
\text { Hematoma no VE }\end{array}$ & Trivial & Não \\
\hline 4 & 17 meses & 8,1 & Trabecular média & 10 & $\mathrm{CoA}$ & 12 & Transfusão, BRE & Não & Não \\
\hline 5 & 4 meses & 5,4 & Trabecular média & 10 & - & 12 & Transfusão & Não & Não \\
\hline 6 & 6 meses & 6,1 & Trabecular média & 8 & $\mathrm{CoA}$ & 10 & Transfusão, BRD & Não & Não \\
\hline 7 & 5 meses & 5,5 & Trabecular média & 14 & - & 16 & $\begin{array}{l}\text { Prolapso do VD } \\
\text { Retirada cirúrgica }\end{array}$ & Discreto & - \\
\hline 8 & 24 meses & 12,3 & Apical & 15 & Pós-bandagem & 16 & - & Não & Não \\
\hline 9 & 3 meses & 4,8 & Trabecular média & 5 & $\mathrm{CoA}$ & 6 & Transfusão & Não & Não \\
\hline
\end{tabular}

$\mathrm{BRD}=$ bloqueio de ramo direito; $\mathrm{BRE}=$ bloqueio de ramo esquerdo; $\mathrm{CIV}=$ comunicação interventricular; CoA = coartação aórtica; $\mathrm{VD}=$ ventrículo direito; $\mathrm{VE}=$ ventrículo esquerdo.

Excetuando-se um paciente (\#3) com duas comunicações interventriculares apicais com dois orifícios no ventrículo esquerdo e múltiplos orifícios de saída no ventrículo direito, todos eram portadores de comunicação interventricular muscular única. Seis comunicações interventriculares eram localizadas na porção trabecular média e o restante, na porção apical. A mediana do maior diâmetro das comunicações interventriculares únicas foi de 10,3 \pm 3,7 mm, variando de $5 \mathrm{~mm}$ a $15 \mathrm{~mm}$.

\section{Procedimentos}

Todos os procedimentos foram completados com sucesso. Não houve dificuldades significativas para identificação do local de punção para permitir um ângulo adequado de progressão dos guias e bainhas. Tampouco houve dificuldades para passagem do guia pela comunicação interventricular para o ventrículo esquerdo. A mediana dos dispositivos utilizados foi de $12 \mathrm{~mm}$. Foram implantadas 10 próteses, porque no paciente portador das comunicações interventriculares múltiplas apicais foram implantadas duas próteses (4 mm e $6 \mathrm{~mm}$ ). Entretanto, em decorrência da posição mais apical de um dos defeitos e da falta de espaço para expansão total do disco direito, a segunda prótese ficou com esse disco discretamente protruso para fora do ventrículo direito, que foi então fixado na parede externa do ventrículo direito com pontos cirúrgicos no 
Pedra CAC, et al. Experiência Multicêntrica na América do Sul no Fechamento Perventricular da Comunicação Interventricular Muscular Congênita. Rev Bras Cardiol Invas. 2009;17(3):386-97.

local (Figura 6). No paciente de 2 anos previamente submetido a bandagem do tronco pulmonar, portador de comunicação interventricular apical de 14-15 mm (Figura 8) com borda posterior deficiente, a prótese de $16 \mathrm{~mm}$ foi fixada na parede do ventrículo direito utilizando-se pequenos pontos transmurais, a fim de evitar um possível prolapso através do defeito (Figura 9). Essa conduta foi tomada pela ocorrência de prolapso da prótese através de um grande defeito em um caso anterior. Sete dos nove pacientes apresentaram oclusão imediata do defeito à ecocardiografia. Como complicações, observamos o aparecimento de pequeno hematoma na parede posterior do ventrículo esquerdo após o implante do segundo dispositivo no paciente com duas comunicações interventriculares apicais, necessitando de pequena plicatura cirúrgica com calço local para reforço da parede. Um paciente apresentou morfologia de bloqueio de ramo direito e outro de bloqueio de ramo esquerdo dentro das primeiras 24 horas após o implante. Um paciente com grande comunicação interventricular de 14 mm (\#7) apresentou bloqueio atrioventricular total, rapidamente revertido com infusão de adrenalina, e agravamento temporário da hipertensão pulmonar já previamente presente. Esse paciente permaneceu com fluxo residual de $2 \mathrm{~mm}$ na porção póstero-inferior da comunicação interventricular, que era deficiente, evoluindo com prolapso da extremidade inferior do disco esquerdo nesse local em direção ao ventrículo direito. Em consequência do aumento do diâmetro do fluxo residual para cerca de $4 \mathrm{~mm}$ e da manutenção do quadro de hipertensão pulmonar após 10 dias, optou-se pela retirada cirúrgica do dispositivo e pela realização de ventriculosseptoplastia com retalho de pericárdio bovino sob circulação extracorpórea. Esse paciente evoluiu bem no pós-operatório, sem fluxos residuais e retorno progressivo das pressões pulmonares para níveis normais com uso de óxido nítrico e sildenafil. Sete
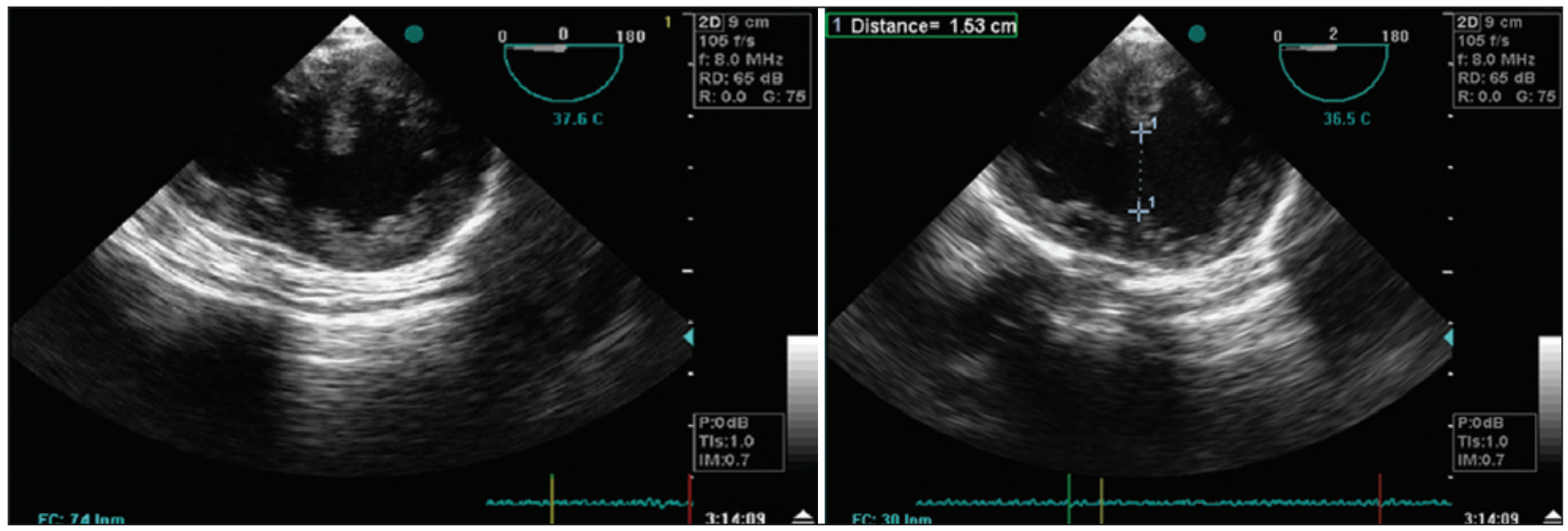

Figura 8 - Grande $(15 \mathrm{~mm})$ comunicação interventricular muscular em região apical em paciente previamente submetido a bandagem da artéria pulmonar. A borda póstero-inferior é curta, quase ausente.
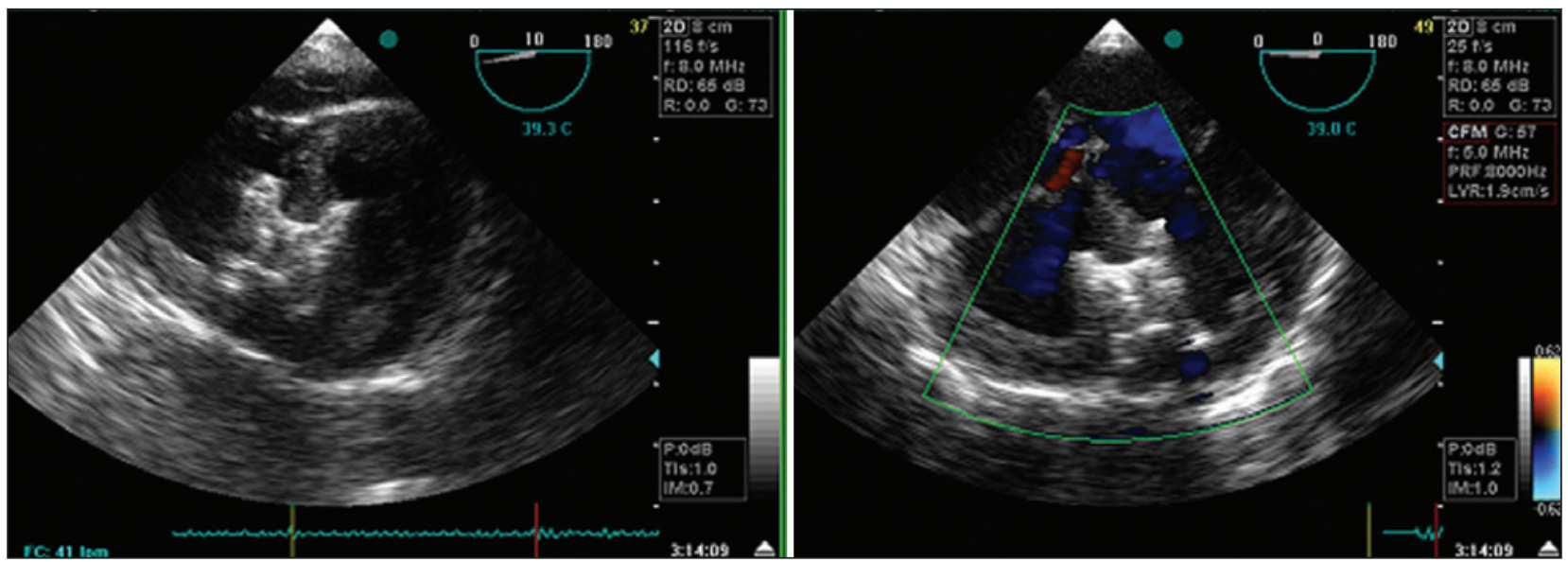

Figura 9 - À esquerda, prolapso da porção inferior do disco esquerdo da prótese de $16 \mathrm{~mm}$ pela borda póstero-inferior deficiente. À direita, após fixação da prótese na parede do ventrículo direito com sutura cirúrgica transmural, a estabilização é obtida. O Doppler em cores mostra oclusão completa do defeito e funcionamento normal das valvas atrioventriculares. 
Pedra CAC, et al. Experiência Multicêntrica na América do Sul no Fechamento Perventricular da Comunicação Interventricular Muscular Congênita. Rev Bras Cardiol Invas. 2009;17(3):386-97.

pacientes necessitaram de transfusão de sangue. O paciente de 45 dias, que estava em ventilação mecânica desde a primeira semana de vida, evoluiu com quadro infeccioso sistêmico relacionado ao cateter e necessitou também de ileostomia, em decorrência de diagnóstico de megacólon congênito.

\section{Seguimento}

A mediana de tempo de internação foi de 7 dias (5-60 dias). Apenas o paciente com as duas comunicações interventriculares apicais ainda apresentava fluxo residual de 1-2 $\mathrm{mm}$ entre as duas próteses à alta hospitalar. Após um ano, observou-se oclusão completa de ambas as comunicações interventriculares. $\mathrm{O}$ seguimento clínico variou de três meses a dois anos, com mediana de um ano. No seguimento, todos os pacientes encontravam-se em ritmo sinusal e apresentavam ventrículo esquerdo com volume diastólico final de dimensões normais à ecocardiografia e função sistólica conservada. Não houve episódios tardios de embolização, endocardite, arritmias, perfurações ou disfunção das valvas atrioventriculares e semilunares. Nos pacientes com coartação da aorta, não houve sinais clínicos nem ecocardiográficos de recoartação.

\section{DISCUSSÃO}

Este estudo demonstrou a exequibilidade da oclusão perventricular da comunicação interventricular muscular em três centros distintos na América do Sul, confirmando a reprodutibilidade do método com diferentes operadores. É importante lembrar que os autores envolvidos neste relato já possuíam larga experiência com outros procedimentos de oclusão percutâneos guiados pela ecocardiografia transesofágica, incluindo o fechamento da comunicação interatrial e das comunicações interventriculares muscular e perimembranosa $a^{12,20,21}$. A familiaridade com as técnicas percutâneas de oclusão, com os dispositivos Amplatzer e com as imagens ecocardiográficas transesofágicas é vital para o sucesso do procedimento por via perventricular, especialmente considerando que as imagens fluoroscópicas não estão disponíveis rotineiramente dentro do centro cirúrgico. Nesse sentido, a realização desse tipo de procedimento em uma sala híbrida pode apresentar vantagens, não só de espaço e de disposição do instrumental mas, especialmente, em casos ocasionais de comunicações interventriculares musculares anteriores, nos quais a angiografia e a combinação de técnicas percutâneas e perventriculares podem ser necessárias para o sucesso do procedimento $^{22}$. Em nosso meio, na América Latina, o estabelecimento desse tipo de sala ainda está longe da nossa realidade, em decorrência de seus altos custos. Excetuando-se esses casos ocasionais, o procedimento é factível, utilizando recursos e equipamentos já disponíveis e relativamente simples, como já demonstrado por outros autores na América do Sul ${ }^{19}$.
Os pacientes aqui relatados formam um grupo especial de alto risco, tanto para a abordagem cirúrgica como para a abordagem percutânea convencionais. São pacientes de baixo peso, com grandes comunicações interventriculares, geralmente desnutridos e com insuficiência cardíaca de difícil controle acompanhada de hipertensão arterial pulmonar e, por vezes, de lesões associadas complicadoras, como a coartação da aorta. Sabe-se que as comunicações interventriculares musculares congênitas constituem-se em desafio técnico ao cirurgião, que tem dificuldade para localizá-las no meio das trabeculações grosseiras do ventrículo direito. Assim, é frequente a ocorrência de fluxos residuais, muitas vezes requerendo reoperações ${ }^{2,22-26}$. As comunicações interventriculares musculares apicais (encontradas em três casos da série aqui apresentada), especialmente as múltiplas, são de abordagem cirúrgica ainda mais difícil, necessitando, por vezes, de ventriculotomia esquerda, que resulta em disfunção ventricular e complicações no pós-operatório ${ }^{2,22-26}$. Tais problemas são exacerbados em pequenos lactentes, como os aqui relatados, o que pode levar a índices de mortalidade não desprezíveis ${ }^{2,22-26}$. Nesses casos, a bandagem da artéria pulmonar pode ser uma opção técnica inicial mais segura, aplicada a um paciente nesta casuística. Além disso, todas as técnicas cirúrgicas, com exceção da bandagem da artéria pulmonar, requerem uso da circulação extracorpórea com suas conhecidas complicações nos diversos sistemas do organismo. Entre as mais temidas encontram-se as crises de hipertensão pulmonar no pósoperatório, em especial em pequenos lactentes com grandes comunicações interventriculares como os aqui apresentados, e, possivelmente, a redução do coeficiente de inteligência e o comprometimento neurológico a longo prazo ${ }^{27-29}$. Adicionalmente, a abordagem cirúrgica necessita de toracotomia, resultando em cicatriz indelével para o resto da vida.

Com a melhoria progressiva dos dispositivos intracardíacos nos últimos dez anos, principalmente os de nitinol de corpo único como são as próteses Amplatzer, a oclusão percutânea da comunicação interventricular muscular tornou-se o método de eleição para a maioria dos pacientes com esse tipo de defeito $^{3-12}$. Apesar de a técnica de implante percutâneo ser relativamente simples, a tensão nos guias e cateteres pode resultar em instabilidade hemodinâmica no laboratório de cateterismo, principalmente em lactentes $^{5,10}$. Em um estudo multicêntrico americano abordando o fechamento percutâneo da comunicação interventricular muscular com próteses Amplatzer, peso inferior a $6 \mathrm{~kg}$ foi considerado fator de risco para ocorrência de complicações graves ${ }^{5}$. Por esse motivo, a técnica perventricular, como a aqui abordada, é de extrema utilidade para o manejo desses defeitos nesse subgrupo de risco de pacientes. Inicialmente relatada por Amin et al. ${ }^{13}$, em 1998, alguns autores subsequentes popularizaram essa forma de abordagem híbrida, que 
Pedra CAC, et al. Experiência Multicêntrica na América do Sul no Fechamento Perventricular da Comunicação Interventricular Muscular Congênita. Rev Bras Cardiol Invas. 2009;17(3):386-97.

viabiliza a oclusão de grandes defeitos em pequenos pacientes como os descritos neste $\operatorname{artigo~}^{14-19}$. A via perventricular permite o uso de bainhas de maior perfil sem prejuízo hemodinâmico e possibilita um ângulo de ataque ideal em direção ao septo interventricular ${ }^{14-19}$. Além disso, outros defeitos associados, como a coartação da aorta, podem ser corrigidos em uma única sessão terapêutica sem a necessidade do uso de circulação extracorpórea, como vimos em quatro pacientes desta experiência e também na literatura ${ }^{14-19}$. Neste relato, em outro paciente, a bandagem previamente colocada no tronco pulmonar foi retirada sem dificuldades após a oclusão perventricular do defeito.

Nesta pequena série de pacientes, o procedimento se mostrou geralmente seguro, com complicações limitadas. O episódio de prolapso para o ventrículo direito possivelmente esteve associado às características do defeito, à escolha inadequada do tamanho do dispositivo ou à estratégia de estabilização insuficiente. Especulamos que um dispositivo de $18 \mathrm{~mm}$ (discretamente superdimensionado) poderia ter proporcionado maior estabilidade ao dispositivo. Entretanto, o espaço restrito dentro do coração poderia ter impedido um implante adequado. Tais casos talvez possam representar uma limitação do método. É importante lembrar que a borda póstero-inferior do defeito neste caso era curta, o que pode ter contribuído para o prolapso. Tendo isso em conta, a fixação externa do dispositivo utilizando suturas cirúrgicas foi empregada em um caso subsequente de grande comunicação interventricular muscular apical com borda posterior limitada, resultando em excelente estabilidade da prótese e oclusão total do defeito. A fixação externa da prótese se fez necessária em outro caso em decorrência da falta de espaço para expansão completa do disco direito perto do ápice. Especula-se se essas suturas utilizadas para a fixação da prótese podem ocasionar arritmias a longo prazo. Nesse sentido, nos casos de comunicações interventriculares muito apicais com espaço restrito para a abertura do disco do lado direito, a prótese Amplatzer para oclusão do canal arterial pode representar melhor opção. O encontro de morfologias de bloqueios de ramos direito e esquerdo neste estudo também foi relatado em outras experiências de fechamento de comunicações interventriculares musculares com próteses Amplatzer ${ }^{30}$. Tais alterações são frequentemente observadas no pós-operatório da correção cirúrgica desses defeitos e não trazem maiores consequências. A ocorrência de bloqueio atrioventricular total após fechamento percutâneo ou perventricular da comunicação interventricular muscular é excepcional $^{8}$, já que os defeitos musculares se encontram distantes do nó atrioventricular. Zahid Amin relata a ocorrência de um caso de bloqueio que necessitou de retirada do dispositivo em 102 casos de sua experiência pessoal na oclusão perventricular de comunicações interventriculares musculares (comunicação pessoal, dados não publicados). A necessidade de transfusão foi uma constante nesta casuística, e esteve, provavelmente, associada à presença de níveis de hematócrito e hemoglobina já limítrofes antes do procedimento, ao baixo peso dos lactentes e aos procedimentos cirúrgicos adicionais em cinco casos. Ressaltamos a necessidade de manejar os cateteres introduzidos pela parede livre do ventrículo direito de forma extremamente cuidadosa para evitar traumas ou perfurações da parede posterior do ventrículo esquerdo (como observado em um paciente desta série). Acreditamos que a técnica de se marcar a bainha introduzida por via perventricular com fios de sutura de acordo com a distância entre a parede anterior do ventrículo direito e a parede posterior do ventrículo esquerdo, como a aqui descrita, é extremamente útil para evitar essa possível complicação. Especula-se que lesões não percebidas na parede posterior do ventrículo esquerdo durante o procedimento podem resultar na formação tardia de aneurismas ventriculares ${ }^{31}$. Além disso, nunca é demais lembrar que os cateteres devem ser sempre limpos com jatos de solução salina, evitando a entrada de ar e a formação de coágulos no sistema, que podem causar complicações tromboembólicas, principalmente no sistema neurológico.

Neste estudo, observamos a alta eficácia do método para oclusão dos defeitos musculares. O único paciente com fluxo residual imediato era portador de comunicações interventriculares apicais múltiplas e apresentou oclusão completa um ano após o implante, em decorrência da endotelização progressiva das próteses. Nesse sentido, os estudos experimentais demonstram que esses dispositivos levam cerca de três a seis meses para serem endotelizados e, por isso, recomenda-se o uso de antiplaquetários durante esse período. Tais taxas de oclusão são, no mínimo, comparáveis às taxas cirúrgicas.

Apesar de a viabilidade econômica desse tipo de procedimento não ter sido o foco deste estudo, o custo associado à utilização de múltiplas próteses para oclusão de comunicações interventriculares musculares múltiplas pode ser um fator limitante em nosso meio. Por outro lado, a aplicação desse método para as comunicações interventriculares musculares únicas provavelmente contribui para recuperação clínica mais rápida e redução de possíveis complicações intrahospitalares, com impacto benéfico sobre os custos.

\section{CONCLUSÃO}

Este estudo demonstrou a exequibilidade, a segurança e a eficácia da oclusão perventricular da comunicação interventricular muscular de etiologia congênita em uma série pequena de lactentes de baixo peso não consecutivos, tratados em múltiplos centros na América do Sul. Mesmo reconhecendo que um maior número de pacientes seria necessário para uma análise mais precisa da segurança do método, é provável que, com o acúmulo de experiência, a incidência de 
Pedra CAC, et al. Experiência Multicêntrica na América do Sul no Fechamento Perventricular da Comunicação Interventricular Muscular Congênita. Rev Bras Cardiol Invas. 2009;17(3):386-97.

complicações, que já é limitada, tendesse a diminuir. Além disso, o tempo de seguimento dos pacientes é ainda curto (mediana de um ano). Entretanto, baseado nos resultados de longo prazo com próteses tipo Amplatzer para oclusão de comunicação interatrial e de outras próteses para oclusão da comunicação interventricular muscular, é improvável que complicações tardias ocorram. Estudos prospectivos e randomizados comparando as técnicas cirúrgicas e perventriculares estão ausentes na literatura e dificilmente serão realizados. Mesmo com essas limitações, acreditamos que os resultados observados neste estudo e em outros disponíveis na literatura nos permitam afirmar que os métodos aqui descritos devam ser escolhidos como os de eleição para o manejo desses casos de alto risco de pequenos lactentes com grandes comunicações interventriculares musculares congênitas.

\section{CONFLITO DE INTERESSES}

Carlos A. C. Pedra é consultor da AGA Medical Corporation (Estados Unidos) e da empresa Bioassist (representante da AGA no Brasil) e recebe honorários em sessões de treinamento, consultoria técnica e palestras ocasionais no Brasil e no exterior. Os demais autores declararam inexistência de conflito de interesses.

\section{REFERÊNCIAS BIBLIOGRÁFICAS}

1. Tynan M, Anderson RH. Ventricular septal defect. In: Anderson $\mathrm{RH}$, Baker EJ, Macartney FJ, Rigby ML, Shinebourne EA, Tynan M, editors. Paediatric cardiology. London: Churchill Livingstone; 2002. p. 983-1014.

2. Kouchoukos NT, Blackstone EH, Doty DB, Hanley FL, Karp RB. Ventricular septal defect. In: Kouchoukos NT, Blackstone EH, Doty DB, Hanley FL, Karp RB, editors. Kirklin/BarratBoyes: Cardiac surgery. Philadelphia: Churchill Livingstone; 2003. p. 850-910.

3. Thanopoulos BD, Tsaousis GS, Konstadopoulou GN, Zarayelyan AG. Transcatheter closure of muscular ventricular septal defects with the Amplatzer ventricular septal defect occluder: initial clinical applications in children. J Am Coll Cardiol. 1999;33(5):1395-9.

4. Hijazi ZM, Hakim F, Al-Fadley F, Abdelhamid J, Cao QL. Transcatheter closure of single muscular ventricular septal defects using the Amplatzer muscular VSD occluder: initial results and technical considerations. Catheter Cardiovasc Interv. 2000;49(2):167-72.

5. Holzer R, Balzer D, Cao QL, Lock K, Hijazi ZM; Amplatzer Muscular Ventricular Septal Defect Investigators. Device closure of muscular ventricular septal defects using the Amplatzer muscular ventricular septal defect occluder: immediate and mid-term results of a U.S. registry. J Am Coll Cardiol. 2004;43(7):1257-63

6. Arora R, Trehan V, Thakur AK, Mehta V, Sengupta PP, Nigam $M$. Transcatheter closure of congenital muscular ventricular septal defect. J Interv Cardiol. 2004;17(2):109-15.

7. Thanopoulos BD, Rigby ML. Outcome of transcatheter closure of muscular ventricular septal defects with the Amplatzer ventricular septal defect occluder. Heart. 2005;91(4):513-6.

8. Carminati $M$, Butera $G$, Chessa $M$, Drago $M$, Negura D, Piazza L. Transcatheter closure of congenital ventricular septal defect with Amplatzer septal occluders. Am J Cardiol. 2005;96(12A):52L-58L.

9. Djer MM, Latiff HA, Alwi M, Samion H, Kandavello G. Transcatheter closure of muscular ventricular septal defect using the Amplatzer devices. Heart Lung Circ. 2006;15(1):12-7.

10. Diab KA, Cao QL, Mora BN, Hijazi ZM. Device closure of muscular ventricular septal defects in infants less than one year of age using the Amplatzer devices: feasibility and outcome. Catheter Cardiovasc Interv. 2007;70(1):90-7.

11. Queiróz FJ, Rossi Filho RI, Ramos S, Esteves C, Queiróz DS, Machado PR, et al. Percutaneous occlusion of interventricular septal defects. Initial experiment. Arq Bras Cardiol. 2005; 85(3):174-9.

12. Pedra CAC, Pedra SRF, Pessotti C, Santana MVT, Jatene I, Shimoda $M$, et al. Fechamento percutâneo da comunicação interventricular muscular congênita. Rev Bras Cardiol Invas. 2008;16(2):218-24.

13. Amin Z, Berry JM, Foker JE, Rocchini AP, Bass JL. Intraoperative closure of muscular ventricular septal defect in a canine model and application of the technique in a baby. J Thorac Cardiovasc Surg. 1998;115(6):1374-6.

14. Bacha EA, Cao QL, Starr JP, Waight D, Ebeid MR, Hijazi ZM. Perventricular device closure of muscular ventricular septal defects on the beating heart: technique and results. J Thorac Cardiovasc Surg. 2003;126(6):1718-23.

15. Bacha EA, Cao QL, Galantowicz ME, Cheatham JP, Fleishman $\mathrm{CE}$, Weinstein SW, et al. Multicenter experience with perventricular device closure of muscular ventricular septal defects. Pediatr Cardiol. 2005;26(2):169-75.

16. Gan C, Lin K, An Q, Tang H, Song H, Lui RC, et al. Perventricular device closure of muscular ventricular septal defects on beating hearts: initial experience in eight children. J Thorac Cardiovasc Surg. 2009;137(4):929-33.

17. Crossland DS, Wilkinson JL, Cochrane AD, d'Udekem $Y$, Brizard CP, Lane GK. Initial results of primary device closure of large muscular ventricular septal defects in early infancy using perventricular access. Catheter Cardiovasc Interv. 2008;72(3):386-91.

18. Celiker A, Ozkutlu S, Erdoðan I, Karagöz T, Doðan OF, Demircin M. Perventricular closure of muscular ventricular septal defect in an infant. Anadolu Kardiyol Derg. 2008;8(4): 312-3.

19. Becker P, Frangini P, Heusser F, Urcelay G, Garay F. New surgical approach to device closure of multiple apical ventricular septal defects. Rev Esp Cardiol. 2004;57(12):1238-40.

20. Pedra CA, Pedra SR, Esteves CA, Pontes SC Jr, Braga SL, Arrieta SR, et al. Percutaneous closure of perimembranous ventricular septal defects with the Amplatzer device: technical and morphological considerations. Catheter Cardiovasc Interv. 2004;61(3):403-10.

21. Pedra CA, Pedra SR, Esteves CA, Chamie F, Christiani LA, Fontes VF. Transcatheter closure of perimembranous ventricular septal defects. Expert Rev Cardiovasc Ther. 2004;2(2):253-64.

22. Diab KA, Hijazi ZM, Cao QL, Bacha EA. A truly hybrid approach to perventricular closure of multiple muscular ventricular septal defects. J Thorac Cardiovasc Surg. 2005; 130(3):892-3.

23. Serraf A, Lacour-Gayet F, Bruniaux J, Ouaknine R, Losay J, Petit J, et al. Surgical management of isolated multiple ventricular septal defects. Logical approach in 130 patients. J Thorac Cardiovasc Surg. 1992;103(3):437-42.

24. Kitagawa T, Durham LA $3^{\text {rd }}$, Mosca RS, Bove EL. Techniques and results in the management of multiple ventricular septal defects. J Thorac Cardiovasc Surg. 1998;115(4):848-56.

25. Hanna B, Colan SD, Bridges ND, Mayer JE, Castaneda AR. 
Pedra CAC, et al. Experiência Multicêntrica na América do Sul no Fechamento Perventricular da Comunicação Interventricular Muscular Congênita. Rev Bras Cardiol Invas. 2009;17(3):386-97.

Clinical and myocardial status after left ventriculotomy for ventricular septal defect closure. J Am Coll Cardiol. 1991;17 Suppl:110A.

26. Wollenek G, Wyse R, Sullivan I, Elliott M, Leval M, Stark J. Closure of muscular septal defects through a left ventriculotomy. Eur J Cardiothorac Surg. 1996;10(8):595-8.

27. Kaltman JR, Jarvik GP, Bernbaum J, Wernovsky G, Gerdes M, Zackai E, et al. Neurodevelopmental outcome after early repair of a ventricular septal defect with or without aortic arch obstruction. J Thorac Cardiovasc Surg. 2006;131(4):792-8.

28. Hövels-Gürish HH, Konrad K, Skorzenski D, Herpertz-Dahlmann B, Messmer BJ, Seghaye MC. Attentional dysfunction in children after corrective cardiac surgery in infancy. Ann Thorac Surg. 2007;83(4):1425-30.
29. Hövels-Gürish HH, Konrad K, Skorzenski D, Minckenberg R, Herpertz-Dahlmann B, Messmer BJ, et al. Long-term behavior and quality of life after corrective cardiac surgery in infancy for tetralogy of Fallot or ventricular septal defect. Pediatr Cardiol. 2007;28(5):346-54.

30. Robinson JD, Zimmerman FJ, De Loera O, Heitschmidt M, Hijazi ZM. Cardiac conduction disturbances seen after transcatheter device closure of muscular ventricular septal defects with the Amplatzer occluder. Am J Cardiol. 2006; 97(4):558-60.

31. Breinholt JP, Rodefeld MD, Hoyer MH. Successful embolization of a left ventricular pseudoaneurysm after perventricular ventricular septal defect device closure. Catheter Cardiovasc Interv. 2009 (in press). 